$\begin{gathered}\text { Revista do Departamento de Geografia } \\ \text { Universidade de São Paulo } \\ \text { www.revistas.usp.br/rdg }\end{gathered}$
V.33 (2017)

\title{
Proposta Metodológica para Análise da Inserção Social na Bacia Hidrográfica do Rio Una em Ibiúna/SP
}

\section{Methodological Proposal for the Analysis of the Social Insertion in Una River Watershed in Ibiúna/SP}

\author{
Darllan Collins da Cunha e Silva \\ Universidade de Sorocaba \\ darllanamb@yahoo.com.br \\ José Luiz Albuquerque Filho \\ Instituto de Pesquisas Tecnológicas/SP \\ albuzelu@ipt.br \\ Renan Angrizani de Oliveira \\ Universidade de Sorocaba \\ renan_angrizani@hotmail.com \\ Roberto Wagner Lourenço \\ UNESP-Sorocaba \\ robertow@sorocaba.unesp.br
}

Recebido (Received): 20/01/2017 DOI: $10.11606 /$ rdg.v33i0.125677
Aceito (Accepted): 31/03/2017
Resumo: Indicadores sociais são importantes mecanismos para a comunicação de informações e provisão de bases sólidas sobre a qualidade de vida dos seres humanos. Nesse estudo elaborou-se um Indicador de Inserção Social (IIS) que integrou informações sociais presentes no censo demográfico da Bacia Hidrográfica do Rio Una (SP), utilizando, em seu cálculo, o método de Análise Hierárquica de Processos (AHP) para valorar as variáveis componentes do indicador e técnicas de geoprocessamento para espacializar os valores do IIS. Este indicador varia de 0 a 1 , onde 1 representa a condição com a melhor qualidade de vida. Ao analisar a bacia hidrográfica do Rio Una foi verificado que não houve valores do IIS acima de 0,50; que poderiam ser considerados como regulares a bom. Aproximadamente 49,4\% da área de estudo apresentam valores de IIS classificados como péssimos e inferiores a 0,25 . Este fato pode estar associado à vocação do município na produção agrícola e na ausência de serviços mais especializados que oferecem uma possibilidade de renda maior, porém exigem um grau de estudo mais elevado, justificados pela baixa renda e a alta taxa de analfabetismo verificada na área de estudo que apresentaram péssimos valores. Através das informações levantadas pode-se concluir que o IIS pode servir como um instrumento importante para a tomada de decisão por gestores públicos e interessados nessa questão.

Palavras-chave: Geoprocessamento; Indicador Social; AHP; Censo Demográfico.

\begin{abstract}
Social indicators are important mechanisms for communicate information and providing solid bases about the human being life quality. In this study, a Social Insertion Indicator (SII) was developed that integrated social information present in the demographic census of the Una River Watershed (SP), using, in its calculation, the Analytic Hierarchy Process (AHP) method to assess the variable components of the indicator and geoprocessing techniques to spatialize the SII values. SII ranges from 0 to 1 , where 1 represents the condition with the best quality of life. When analyzing the Una River Watershed, it was verified that there were no SII values above 0.50, which could be considered as regular to good. Approximately $49.4 \%$ of the study area presented SII values classified as poor and lower than 0.25 . This fact may be associated with the municipality's vocation in agricultural production and in the absence of more specialized services that offer a higher income possibility, but require a higher degree, justified by the low income and the high illiteracy rate in the area of study that presented poor values. Through the information gathered it can be concluded that SII can serve as an important tool for decision-making by public managers and those interested in this issue.
\end{abstract}

Keywords: Geoprocessing; Social Indicator; AHP; Demographic census. 


\section{INTRODUÇÃO}

Indicador é um instrumento que possibilita obter informações quantificadas sobre o estado de um fenômeno observado. Por isso, os indicadores são considerados importantes mecanismos de suporte à tomada de decisões envolvendo questões socioambientais (VAN BELLEN, 2006; SICHE et al., 2007).

Portanto, indicadores sinalizam a situação de um fenômeno, pois são valores estáticos, isto é, dimensionam o momento atual, entretanto, o meio ambiente é um sistema dinâmico, assim, os índices têm uma potencialidade de previsão para um curto período de tempo (SICHE et al., 2007).

Segundo Lourenço et al. (2015), os indicadores socioambientais são importantes ferramentas nos processos de gestão, especialmente quando aplicados às bacias hidrográficas, pois representam um importante avanço na gestão dessa unidade de planejamento socioambiental.

Deponti et al. (2002) citam que a modelagem de indicadores deve estar diretamente relacionada com a avaliação e monitoramento do sistema estudado, podendo ser usados dados não numéricos, porém estes necessitam serem traduzidos em itens mensuráveis, isto é, quantificáveis. Guimarães e Magrini (2008) ressaltam que os indicadores devem apresentar uma interface objetiva e comunicativa para serem usados, além de apresentarem custo e coleta de dados viáveis para sua aplicação. Desta forma, passam a serem úteis como ferramentas de gestão, pois direcionam de forma eficiente as ações dos gestores.

Vários estudos utilizaram indicadores sociais para avaliar as desigualdades sociais presentes em uma determinada região, como os de Bata e Mariano (2015), que analisaram a vulnerabilidade socioambiental nas aldeias de Nanhupo e Nséue no distrito de Monte Puez (Moçambique), onde é realizado o garimpo de pedras preciosas, de Gonçalves et al. (2014) que verificaram a vulnerabilidade socioambiental decorrente do processo de urbanização na Amazônia no município de Porto Velho (Brasil), por meio da construção de um indicador que integrasse a dimensão social, infraestrutura habitacional, exposição ambiental e de saúde, e de Santana et al. (2014) que construíram um indicador que permitisse contribuir com a identificação e avaliação na saúde da população frente aos impactos socioambientais analisados no município de Amadora (Portugal).

No presente estudo foi elaborado um Indicador de Inserção Social (IIS) representado por um valor numérico, onde o peso de cada variável foi calculado pelo método da Análise Hierárquica de Processos (AHP). Este indicador possibilita o reconhecimento das desigualdades e condições sociais dos residentes na Bacia Hidrográfica do Rio Una (SP). Além disso, é um importante instrumento para avaliar espacialmente a distribuição desses dados, pois utiliza em seu cálculo, dados do censo demográfico e técnicas de geoprocessamento.

\section{MATERIAIS E MÉTODOS}

A área de estudo é denominada de Bacia Hidrográfica do Rio Una e está localizada no interior do Estado de São Paulo no Município de Ibiúna que possui uma população de 73.309 habitantes com 55,7\% dos habitantes residentes na zona rural, devido a agricultura ser a principal atividade econômica do município (SEADE, 2017). O Rio Una juntamente com os Rios Sorocabuçu e o Sorocamirim desembocam no reservatório de Itupararanga, o qual possui grande importância regional, sendo o principal manancial de captação de água para o município de Sorocaba e região, além de ser utilizado como fonte de irrigação para propriedades agrícolas nos arredores (SILVA et al., 2016).

A Bacia Hidrográfica do Rio Una (Figura 1) foi escolhida por estar sob influência de diversas atividades antrópicas que estão fragmentando a paisagem e ignorando a aptidão do uso e cobertura do solo, o que possibilita trabalhar diferentes unidades da paisagem (SILVA et al., 2016). Além disso, não foram encontrados estudos científicos que abordem as questões sociais na bacia hidrográfica, portanto, este estudo deve contribuir com a construção de informações georreferenciadas para as comunidades sob influência dessa área.

As cartas topográficas do Instituto Geográfico e Cartográfico (IGC) na escala 1:10.000 (folhas: 094/096; 095/095; 095/096; 096/095; 096/096; 097/095; 097/096; 097/094 e 098/095) foram utilizadas para a delimitação da Bacia Hidrográfica do Rio Una e digitalizadas no ArcGIS 10.1. Esse limite da bacia hidrográfica foi utilizado juntamente com a base cartográfica do Censo Demográfico de 2010 (IBGE, 2012) para extrair os setores censitários presentes na área de estudo. 
O Indicador de Inserção Social (IIS) proposto neste estudo foi gerado a partir de informações socioambientais classificadas em termos de variáveis que buscam refletir as condições materiais e sociais da população residente na Bacia Hidrográfica do Rio Una com a finalidade de reconhecer as desigualdades sociais presentes na área de estudo.

As variáveis do IIS foram extraídas do banco de dados do Censo Demográfico de 2010 (IBGE, 2012) para o município de Ibiúna contemplando todos os setores censitários presentes no município. Neste estudo foram utilizados os dados de 42 setores censitários presentes na Bacia Hidrográfica do Rio Una, enquanto que as tabelas empregadas no desenvolvimento do IIS foram tratadas e manipuladas com o auxílio do software Microsoft Office Excel 2010.

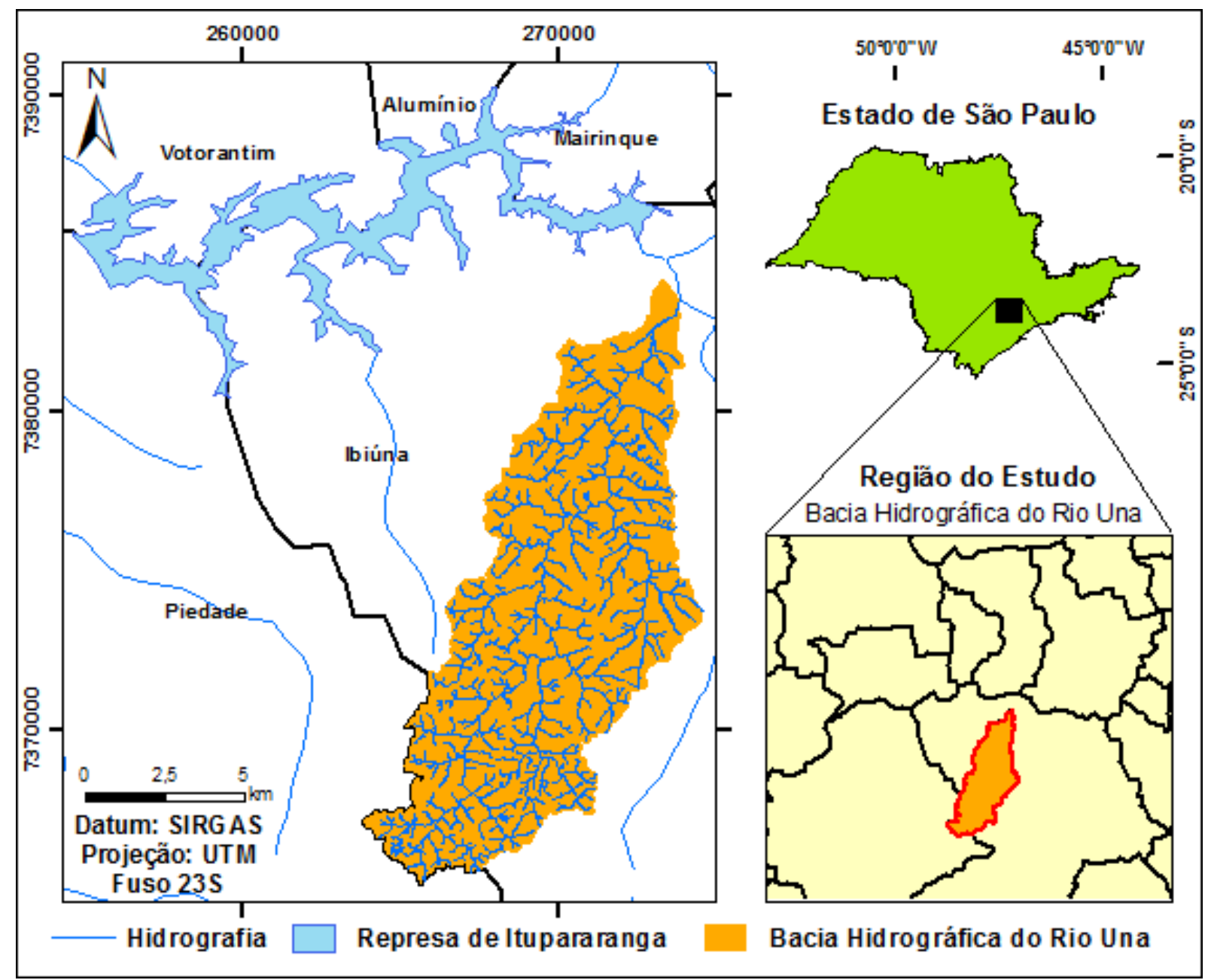

Figura 1. Localização da Bacia Hidrográfica do Rio Una. Fonte: Silva et al. (2016)

Os setores censitários que extrapolassem o limite da Bacia Hidrográfica do Rio Una foram recortados acompanhando os limites da área de estudo e seus dados ponderados por uma razão entre a área do setor censitário recortado e inserido na Bacia Hidrográfica pela área total desse setor censitário sem recortes.

Para a construção do IIS foram utilizadas as variáveis condições de ocupação dos domicílios, renda, taxa de alfabetização (maiores de 15 anos) e taxa dos responsáveis pelos domicílios particulares permanentes com mais de 18 anos.

Os pesos de cada uma das variáveis que compõem IIS foi obtido pelo particionamento do intervalo de 0 a 1 em 4 valores compreendendo os extremos desse intervalo e o primeiro e terceiro quartil do referido intervalo. A Tabela 1 apresenta a atribuição dos pesos de cada um dos componentes do IIS, sendo que as variáveis foram classificadas ainda, segundo o estado da condição dos ocupantes do domicilio, em ótima, boa, regular e ruim. 
Tabela 1. Atribuição de pesos para cada uma das variáveis do IIS

\begin{tabular}{|c|c|c|}
\hline VARIÁVEIS & CONDIÇÕES & PESOS \\
\hline \multirow{4}{*}{$\begin{array}{l}\text { Condição de ocupação } \\
\text { do domicílio }(\mathrm{COD})^{1}\end{array}$} & $\begin{array}{l}\text { Condição ótima: domicílios particulares permanentes próprios (quitados ou em } \\
\text { aquisição). }\end{array}$ & 1 \\
\hline & Condição boa: domicílios particulares permanentes alugados. & 0,75 \\
\hline & $\begin{array}{l}\text { Condição regular: domicílios particulares permanentes cedidos (por empregador ou } \\
\text { outra forma). }\end{array}$ & 0,25 \\
\hline & $\begin{array}{l}\text { Condição ruim: domicílios particulares improvisados, domicílios coletivos e outras } \\
\text { condições de ocupação. }\end{array}$ & 0 \\
\hline \multirow{4}{*}{ Renda $(\mathrm{REN})^{1}$} & Condição ótima: 3 ou mais salários mínimos per capita. & 1 \\
\hline & Condição boa: entre 2 e $<3$ salários mínimos per capita. & 0,75 \\
\hline & Condição regular: entre 1 e $<2$ salários mínimos per capita. & 0,25 \\
\hline & Condição ruim: entre 0 e $<1$ salário mínimo per capita. & 0 \\
\hline \multirow{4}{*}{$\begin{array}{l}\text { Taxa de alfabetização } \\
\qquad(\mathrm{ALF})^{1,2}\end{array}$} & Condição ótima: Taxa de pessoas alfabetizadas com 15 anos ou mais igual a 100\%. & 1 \\
\hline & $\begin{array}{l}\text { Condição boa: Taxa de pessoas alfabetizadas com } 15 \text { anos ou mais, maior ou igual a } \\
90 \% \text { e menor que } 100 \% \text {. Ressalta-se que a taxa média do Brasil é 90,4\% (PNUD, } \\
\text { 2014). }\end{array}$ & 0,75 \\
\hline & $\begin{array}{l}\text { Condição regular: Taxa de pessoas alfabetizadas com } 15 \text { anos ou mais, maior ou } \\
\text { igual a } 70 \% \text { e menor que } 90 \% \text {. }\end{array}$ & 0,25 \\
\hline & $\begin{array}{l}\text { Condição ruim: Taxa de pessoas alfabetizadas com } 15 \text { anos ou mais, menor que } \\
70 \% \text {. }\end{array}$ & 0 \\
\hline \multirow{4}{*}{$\begin{array}{l}\text { Taxa de Responsáveis } \\
\text { pelo domicílio } \\
\text { (RESP) })^{1}\end{array}$} & $\begin{array}{l}\text { Condição ótima: Taxa de pessoas responsáveis pelo domicílio com } 18 \text { anos ou mais } \\
\text { igual a } 100 \% \text {. }\end{array}$ & 1 \\
\hline & $\begin{array}{l}\text { Condição boa: Taxa de pessoas responsáveis com } 18 \text { anos ou mais, maior ou igual a } \\
90 \% \text { e menor que } 100 \% \text {. }\end{array}$ & 0,75 \\
\hline & $\begin{array}{l}\text { Condição regular: Taxa de pessoas responsáveis com } 18 \text { anos ou mais, maior ou } \\
\text { igual a } 70 \% \text { e menor que } 90 \% \text {. }\end{array}$ & 0,25 \\
\hline & $\begin{array}{l}\text { Condição ruim: Taxa de pessoas responsáveis com } 18 \text { anos ou mais, menor que } \\
70 \% \text {. }\end{array}$ & 0 \\
\hline
\end{tabular}

${ }^{1}$ Esses dados foram disponibilizados pelo IBGE apenas para domicílios particulares permanentes, excluindo-se a condição dos domicílios improvisados e coletivos.

${ }^{2}$ IBGE considerou como alfabetizada a pessoa capaz de ler e escrever um bilhete simples no idioma que conhecesse e analfabeta a pessoa que não sabe ler ou escrever e a que apenas assina o próprio nome.

O valor final por setor censitário para as variáveis estudadas de Condição de ocupação do domicílio - COD e Renda - REN foram obtidos por meio de uma média ponderada em função do número de domicílios em cada condição de acordo com a Equação 1:

$$
\operatorname{Var}=\frac{\mathrm{Co} \times \mathrm{No}+\mathrm{Cb} \times \mathrm{Nb}+\mathrm{Crg} \times \mathrm{Nrg}+\mathrm{Crm} \times \mathrm{Nrm}}{\mathrm{No}+\mathrm{Nb}+\mathrm{Nrg}+\mathrm{Nrm}}
$$

Sendo:

Var é a variável estudada que pode ser a COD ou REN;

Co é o peso da condição ótima;

Cb é o peso da condição boa;

Crg é o peso da condição regular;

Crm é o peso da condição ruim;

No é o número de domicílios classificados como condição ótima;

$\mathrm{Nb}$ é o número de domicílios classificados como condição boa;

Nrg é o número de domicílios classificados como condição regular;

Nrm é o número de domicílios classificados como condição ruim. 
Para o cálculo da taxa de alfabetização foi considerado o critério estabelecido no Índice de Desenvolvimento Humano (IDH) pelo Programa das Nações Unidas para o Desenvolvimento (PNUD) que fixou a idade máxima de 15 anos para que uma pessoa já esteja alfabetizada, isto é, seja capaz de ler e escrever um bilhete simples (PNUD, 2014). Daí a opção por se medir essa capacidade na população com 15 anos ou mais de idade. A taxa de alfabetização (ALF) foi calculada por setor censitário de acordo com a Equação 2:

$$
A L F=\frac{P A L F}{N M S C} \times 100 \%
$$

Sendo:

ALF é a taxa de alfabetização em porcentagem;

PALF é o número de pessoas com 15 anos ou mais alfabetizadas no setor censitário;

NMSC é o número de pessoas com 15 anos ou mais residentes no setor censitário.

Para o cálculo da taxa de responsáveis pelo domicilio foi considerado como idade mínima 18 anos para exercer essa função, uma vez que, com 18 anos a pessoa adquire a maioridade de acordo com a legislação brasileira e espera-se que tenha maturidade suficiente para gerir uma família conforme é previsto na Lei $\mathrm{n}^{\circ}$. 10.406 (BRASIL, 2002). A taxa de responsáveis pelo domicilio (RESP) foi calculada de acordo com a Equação 3:

$$
R E S P=\frac{P R D}{N P R D S C} \times 100 \%
$$

Sendo:

RESP é a taxa de pessoas responsáveis por domicilio em porcentagem;

PRD é o número de pessoas com 18 anos ou mais responsáveis pelo domicilio no setor censitário;

NPRDSC é o número total de pessoas responsáveis pelo domicilio no setor censitário.

Para quantificar o peso de cada variável do IIS foi aplicado o método proposto por Saaty (1991) de Análise Hierárquica de Processos (AHP). O emprego deste método nesse estudo deve-se à minimização dos critérios subjetivos para a ponderação entre as variáveis (OLIVEIRA et al., 2016).

As comparações pareadas das variáveis do IIS foram realizadas com base em uma escala de prioridades padrão, própria da Análise Hierárquica de Processos. Para isso, foi gerada uma matriz de comparação expressa por meio da relação de importância de uma determinada variável (linha) frente à outra variável (coluna). Após esse processo foi calculada a matriz ponderada, que consiste na divisão de cada elemento da coluna pela soma dos valores da própria coluna da matriz de comparação.

Após a elaboração da matriz ponderada obteve-se os pesos normalizados para cada variável do IIS através do cálculo da média dos valores de cada linha da matriz ponderada. Em seguida foi calculado o índice de consistência (IC) para verificar os pesos obtidos (SAATY, 1991). Ainda segundo o autor, se o IC for menor do que 0,1 ; então há consistência para prosseguir com os cálculos do AHP, caso contrário, recomenda-se que os julgamentos sejam refeitos até que haja consistência. Além disso, foi calculado a Razão de Consistência (RC), que é calculada dividindo-se o IC pelo Índice Randômico (IR), o qual varia de acordo com o tamanho $n$ da amostra estabelecido por Saaty (1991). 
Após os cálculos dos pesos pelo método AHP e dos valores das variáveis integrantes do IIS, foi calculado o valor final do IIS para cada setor censitário através da Equação 4:

$$
I I S=\sum_{i=1}^{4} \operatorname{Var}(i) \times p(i)
$$

Sendo:

Var(i) é o valor das variáveis (COD, REN, ALF e RESP) para um determinado setor censitário;

p(i) é o valor do peso normalizado das variáveis calculadas pelo método AHP.

Os valores finais do IIS para cada setor censitário foram geocodificados no ArcGIS 10.1 para alimentar o banco de dados dos setores censitários presentes na Bacia Hidrográfica do Rio Una.

\section{RESULTADOS E DISCUSSÃO}

A Figura 2 mostra os mapas das variáveis utilizadas e extraídas do banco de dados do Censo Demográfico de 2010 (IBGE, 2012) para o município de Ibiúna na elaboração do IIS com seus valores finais por setor censitário divididos em quartis ou quatro partes iguais $(0$ a 0,$25 ; 0,25$ a 0,$50 ; 050$ a 0,75 e 0,75 a 1$)$ para todas as variáveis componentes deste índice.

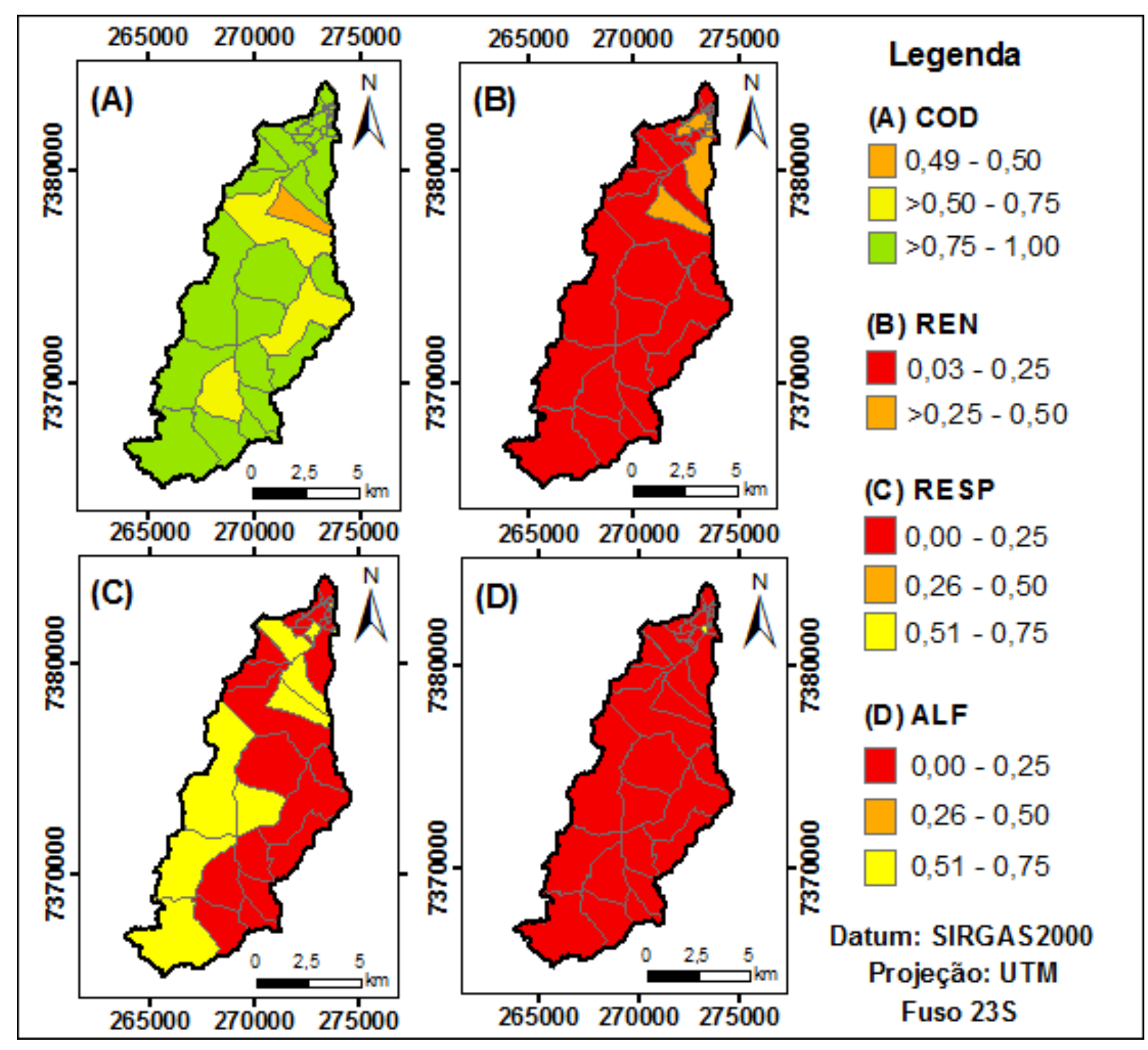

Figura 2. Mapas das variáveis utilizadas na construção do IIS 
A Tabela 2 apresenta a distribuição em porcentagem das variáveis componentes do IIS pelos intervalos de valores apresentados na Figura 2.

Tabela 2. Distribuição (em \%) das variáveis do IIS por intervalos de valores

\begin{tabular}{ccccc}
\hline Intervalo & REN & RESP & ALF & COD \\
\hline 0 a 0,25 & $78,6 \%$ & $76,2 \%$ & $97,6 \%$ & $0,0 \%$ \\
0,25 a 0,5 & $21,4 \%$ & $0,0 \%$ & $0,0 \%$ & $2,4 \%$ \\
0,5 a 0,75 & $0,0 \%$ & $23,8 \%$ & $2,4 \%$ & $14,3 \%$ \\
0,75 a 1 & $0,0 \%$ & $0,0 \%$ & $0,0 \%$ & $83,3 \%$ \\
\hline
\end{tabular}

Para a variável COD, somente um setor censitário apresentou valor inferior a 0,5; e aproximadamente 83,3\% dos domicílios apresentam valores superiores a 0,75 ; isto indica que a maior parte dos domicílios presentes na área de estudo estão quitados ou em processo de aquisição segundo classificação utilizada pelo IBGE (2012).

Isto é importante, uma vez que, as pessoas com domicílios quitados possuem, em sua maioria, uma oportunidade maior de investir seus recursos financeiros no crescimento pessoal e profissional da família do que aqueles que não se encontram nessa situação (ROSINKE et al., 2011; ALVES; CAVENAGHI, 2016; MATOS et al., 2016).

Em relação a variável REN, verifica-se que quase $79 \%$ da população residente nesta área de estudo, sobrevivem com menos de um salário mínimo per capita, indicando a vulnerabilidade social dos residentes na Bacia Hidrográfica do Rio Una. Ainda, corroborando com esta afirmação, é possível verificar no mapa da Figura 2 que não há nenhum setor censitário que apresente uma condição classificada como ótima.

A variável RESP, correspondente a taxa de pessoas responsáveis pelo domicílio com 18 anos ou mais, está bem distribuída na área de estudo, com valores inferiores a 0,75 ; indicando que não há nenhum setor censitário que apresente todos os responsáveis pelo domicílio com idade superior a 18 anos, além disso, os que apresentam menos de $70 \%$ dos responsáveis com mais de 18 anos correspondem a $76 \%$ aproximadamente do total.

Portanto, é esperado que os responsáveis pelo domicílio possuam 18 anos ou mais, pois se espera que todos os responsáveis pelo domicílio tenham obtido uma oportunidade de concluir o ensino médio antes de se tornar o responsável pelo sustento de sua família. Em casos contrários é relatado um aumento da desigualdade social, pois o responsável pela família dificilmente tem um emprego com boa remuneração devido à baixa escolaridade e, consequentemente, na maioria dos casos, não consegue sustentar sua família e voltar a estudar (ALVES, 2006; REMES e MARTIKAINEN, 2015; ALVES e CAVENAGHI, 2016).

ALF é a única variável que apresentou quase $100 \%$ dos seus dados em uma única classe de valores e, também, é a única que tem quase todos os valores presentes no primeiro quartil, isto é, pior classe de valores. Ou seja, o nível de analfabetismo presente nos setores censitários apresenta uma taxa de pessoas alfabetizadas com 15 anos ou mais, menor que 70\%, bem inferior à média brasileira que é de 90,4\% (PNUD, 2014). Portanto, é a variável mais preocupante da Bacia Hidrográfica e que necessita de atenção especial por parte dos gestores públicos.

Para quantificar o peso de cada variável do IIS foi aplicado o método de AHP. Para tanto, foi estabelecida uma matriz de comparação pareada entre as quatro variáveis componentes do IIS por comparações par a par, a partir do julgamento da intensidade de importância de cada variável conforme é apresentado na Tabela 3. 
Tabela 3. Matriz de comparações pareadas das variáveis componentes do IIS

\begin{tabular}{c|cccc}
\hline Variáveis do IIS & REN & ALF & RESP & COD \\
\hline REN & 1 & 1 & 3 & 5 \\
ALF & 1 & 1 & 3 & 5 \\
RESP & $1 / 3$ & $1 / 3$ & 1 & 3 \\
COD & $1 / 5$ & $1 / 5$ & $1 / 3$ & 1 \\
\hline
\end{tabular}

A Tabela 4 apresenta os pesos normalizados para cada uma das variáveis componentes do IIS, bem como o valor calculado do autovalor máximo $\left(\lambda_{\max }\right)$ utilizado na obtenção do índice de consistência (IC).

Tabela 4. Pesos normalizados das variáveis componentes do IIS

\begin{tabular}{cccc}
\hline Variáveis do IIS & Pesos normalizados(p) & Ap & Ap/p \\
\hline REN & 0,39 & 1,58 & 4,07 \\
ALF & 0,39 & 1,58 & 4,07 \\
RESP & 0,15 & 0,62 & 4,03 \\
COD & 0,07 & 0,28 & 4,01 \\
\hline & & Média $(\lambda$ Max $)$ & 4,04 \\
\hline
\end{tabular}

O valor do IC calculado para avaliar a consistência dos pesos atribuídos às variáveis componentes do IIS pelo método da AHP foi de 0,015; isto é, abaixo de 0,1 e, portanto, há consistência nos valores obtidos. Para reforçar ainda mais a avaliação da consistência dos dados, foi calculado a razão de consistência (RC) e o valor obtido foi de 0,016; ou seja, abaixo de 0,1; indicando que os pesos normalizados calculados apresentam consistência. As variáveis que apresentaram os maiores pesos foram REN e a ALF com pesos iguais a 0,39; enquanto que, a variável COD apresentou o menor peso e igual a 0,07 como pode ser visto na Tabela 4.

A partir disso, foi calculado o valor do Indicador de Inserção Social (IIS) para cada setor censitário e dividido em quartis para serem geoespacializados como é mostrado no mapa da Figura 3.

Os piores valores para o IIS encontram-se distribuídos ao longo da Bacia Hidrográfica, porém com maior presença para a região central e sul, isto é, onde há a maior concentração de atividades agrícolas. Não houve bons resultados para os valores calculados do IIS, possivelmente, devido aos péssimos valores encontrados para a taxa de alfabetização e renda (Tabela 2), que são as variáveis de maior peso no cálculo do IIS, ou seja, a população residente nessa área carece de maior acesso à educação e fonte de renda.

Para a maior parte dos setores censitários estudados, a principal fonte de renda da população provém da agricultura de subsistência, isto é, de pequenas propriedades agrícolas. Porém, mesmo ao norte da Bacia Hidrográfica, onde reside maior parte da população e está localizada a área urbana do município, os valores de IIS foram baixos como podem ser vistos na Figura 3.

Os valores do IIS distribuídos por setores censitários na bacia hidrográfica não apresentaram valores acima de 0,50; ou seja, não alcançaram nem 50\% do valor máximo admissível pelo índice. Além disso, aproximadamente $49,4 \%$ da área de estudo apresentam valores de IIS que podem ser classificados como péssimos $(0,25)$. Este fato pode estar associado às principais vagas de emprego disponíveis no município que estão alocadas na produção agrícola (22\%) e no comércio e serviços (61\%), isto é, há baixa oferta de empregos com serviços mais especializados que exigem um grau de estudo mais elevado e renda maior, o que pode explicar os baixos valores encontrados para a taxa de alfabetização e renda dos residentes da área de estudo (SEADE, 2017).

O IIS foi capaz de captar essa desigualdade e transformá-la numericamente, o que possibilita utilizar esse índice em outras áreas para comparar os resultados, além de ser um instrumento que possibilita orientar os gestores públicos quanto às áreas que mais necessitam de investimentos e aquelas que necessitam de maior atenção do governo para atrair novos investidores. 


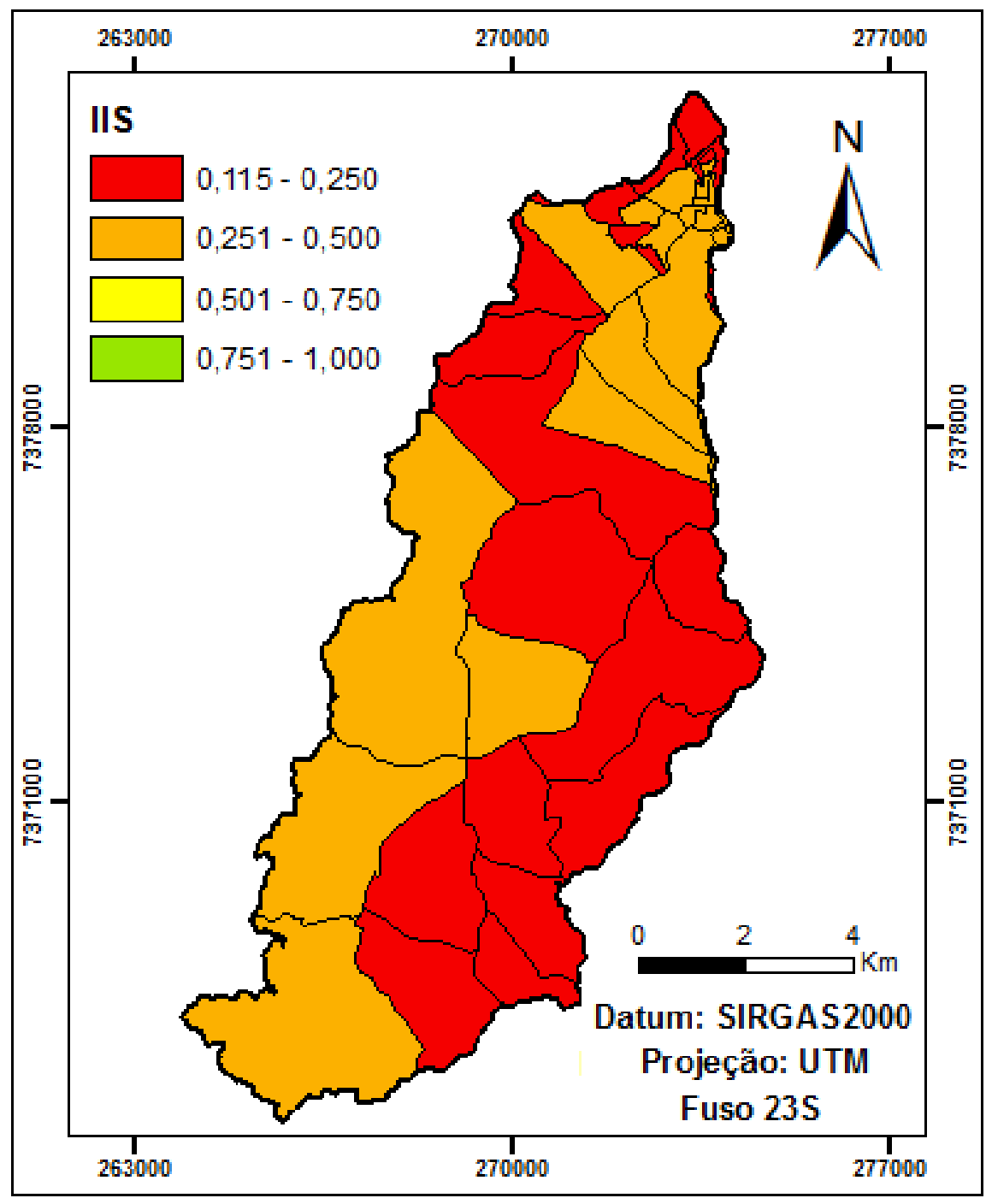

Figura 3. Distribuição dos valores do IIS por setores censitários

\section{CONCLUSÕES}

A partir dos resultados foi possível concluir que as desigualdades sociais estão presentes em toda a área de estudo que apresentou péssimos valores de IIS, devido principalmente à baixa renda da população e à alta taxa de analfabetismo presente na área de estudo. Conclui-se que o IIS é sensível o suficiente para apontar os indicadores que mais influenciam no seu valor e aqueles que necessitam de uma gestão prioritária.

O valor médio do IIS para a bacia hidrográfica foi de 0,28 ; podendo ser classificado como ruim $(0,26$ a 0,50$)$ quando comparado aos valores estabelecidos pelo IGAM (2005) para o Índice de Qualidade da Água (IQA). Há necessidade de investimentos na área da educação, especialmente devido à taxa de responsáveis por domicílios menores que 18 anos ser alta, o que reflete negativamente na estrutura familiar e no aperfeiçoamento profissional do responsável pela família e, consequentemente, na renda familiar.

Através das informações levantadas neste estudo, pode-se concluir que o IIS é uma importante ferramenta que provém da agregação de alguns indicadores sociais que permitem interpretar numericamente a realidade social da Bacia Hidrográfica do Rio Una, enfocando os residentes dessa área e, portanto, é uma alternativa válida para servir como um instrumento de tomada de decisão para gestores públicos e interessados nessa questão. 


\section{REFERÊNCIAS}

ALVES, H. P. F. Vulnerabilidade socioambiental na metrópole paulistana: uma análise sociodemográfica das situações de sobreposição espacial de problemas e riscos sociais e ambientais. Revista Brasileira de Estudos de População, v. 23, n. 1, p. 43-59, 2006.

ALVES, J. E. D.; CAVENAGHI, S. Déficit habitacional, famílias conviventes e condições de moradia. Séries Demográficas, v. 3, p. 257-286, 2016.

BRASIL. Lei n. 10.406, 10 de janeiro de 2002. Código Civil. Diário Oficial da União, Rio de Janeiro, 11 jan. 2002.

BATA, E. J.; MARIANO, Z. F. A. Vulnerabilidade Socioambiental no contexto da exploração das pedras preciosas e semipreciosas em Namanhumbir, distrito de Montepuez (Moçambique), entre 2004 e 2011. Revista do Departamento de Geografia, v. 29, p. 34-58, 2015.

DEPONTI, C. M.; ECKERT, C.; AZAMBUJA, J. L. B. Estratégia para construção de indicadores para avaliação da sustentabilidade e monitoramento de sistemas. Agroecologia e Desenvolvimento Rural Sustentável, v. 3, n. 4, p. 44-52, 2002.

GONCALVES, K. S.; SIQUEIRA, A. S. P.; CASTRO, H. A.; HACON, S. S. Indicador de vulnerabilidade socioambiental na Amazônia Ocidental. O caso do município de Porto Velho, Rondônia, Brasil. Ciência \& Saúde Coletiva, v. 19, n. 9, p. 3809-3818, 2014.

GUIMARÃES, L. T.; MAGRINI, A. A proposal of indicators for sustainable development in the management of river basins. Water Resources Management, v. 22, n. 9, p. 1191-1202, 2008.

IBGE - Instituto Brasileiro de Geografia e Estatística. Base Estatcart de Informações do Censo Demográfico 2010: Resultados do Universo por Setor Censitário. Rio de Janeiro, IBGE, 2012. CD-ROM.

IGAM, Instituto Mineiro de Gestão das Águas. Sistema de Cálculo de Índice de Qualidade de Água (SCQA) - Estabelecimento das Equações do índice de Qualidade das Águas (IQA). Belo Horizonte: IGAM, 2005. $16 \mathrm{p}$.

LOURENÇO, R. W.; SILVA, D. C. C.; MARTINS, A. C. G.; SALES, J. C. A.; ROVEDA, S. R. M. M.; ROVEDA, J. A. F. Use of fuzzy systems in the elaboration of an anthropic pressure indicator to evaluate the remaining forest fragments. Environmental Earth Sciences, v. 73, p. 1-8, 2015.

MATOS, R. E. S.; LOBO, C. F. F.; CHAVES, A. A. Evolução recente da propriedade domiciliar no Brasil. Investigaciones Geográficas, n. 91, p. 124-136, 2016.

OLIVEIRA, R. A.; SILVA, D. C. C.; SIMONETTI, V. C.; STROKA, E. A. B.; SABONARO, D. Z. Proposição de Corredor Ecológico entre duas Unidades de Conservação na Região Metropolitana de Sorocaba. Revista do Departamento de Geografia, v. 32, p. 61-71, 2016.

PNUD - Programa das Nações Unidas para o Desenvolvimento. Relatório de Desenvolvimento Humano 2014. Sustentar o Progresso Humano: Reduzir as Vulnerabilidades e Reforçar a Resiliência. Nova Iorque: PNUD, 2014.

REMES, H.; MARTIKAINEN, P. Young adult's own and parental social characteristics predict injury morbidity: a register-based follow-up of 135000 men and women. BMC Public Health, v. 15, n. 1, p. 429, 2015.

ROSINKE, J. G.; HECK, C. R.; DALFOVO, W. C. T.; RUSCHEINSKY, A. Efeitos sociais e econômicos para o desenvolvimento local através das contribuições do Programa Bolsa Família no município de Sinop-MT no período de 2004 a 2009. Interações, v. 12, n. 1, p. 77-88, 2011.

SAATY, T. L. Método de Análise Hierárquica. São Paulo: McGraw-Hill Publisher, 1991. 367 p.

SANTANA, P.; COSTA, C.; LOUREIRO, A. Os Sistemas de Informação Geográfica e o Planejamento Urbano Saudável na Amadora. Revista do Departamento de Geografia, v. spe, p. 368-389, 2014.

SEADE - Fundação Sistema Estadual de Análise de Dados. Informação dos Municípios Paulistas (IMP). Disponível em: < http://www.imp.seade.gov.br/frontend/\#/>. Acesso em: 19 Jan. 2017.

SICHE, R.; AGOSTINHO, F.; ORTEGA, E.; ROMEIRO, A. Índices versus indicadores: precisões conceituais na discussão da sustentabilidade de países. Ambiente \& Sociedade, v. 10, n. 2, p. 137-148, 2007. 
SILVA, D. C. C.; ALBUQUERQUE FILHO, J. L.; SALES, J. C. A.; LOURENÇO, R. W. Uso de indicadores morfométricos como ferramentas para avaliação de bacias hidrográficas. Revista Brasileira de Geografia Física, v. 9, n. 2, p. 221-217, 2016.

VAN BELLEN, H. M. Indicadores de Sustentabilidade: Uma Análise Comparativa. 2. ed. Rio de Janeiro: FGV, 2006. 